\title{
Challenges in Managing Diffusion of Telemedicine Technology in South Africa
}

\author{
Michael Ogembo Kachieng'a
}

University of Pretoria, Lynnwood Road, Hatfield, Pretoria South Africa

\begin{abstract}
Technological progresses in the information, communication and telecommunication (ICT) industries and innovations telemedia industry have made telemedicine an increasingly viable health care delivery choice for emerging economies like South Africa. Simply stated telemedicine is the use of ICT technologies in conjunction with telemedia systems to provide health care services when distance separates the health professionals and patients. The study examines the constraints of utilisation of telemedicine in the broader health care delivery value chain. This paper discusses the challenges of managing diffusion of telemedicine technology in the rural areas of South Africa. Specific telemedicine project installations are discussed and recommendations provided to guide policy makers and health planners in South Africa.
\end{abstract}

Keywords Technology, Telecommunication, Health, Innovation

\section{Introduction}

Medical practice and health care services depend on effective diffusion of health technology to meet their variety of needs. They use technology to gather information necessary for appropriate diagnosis; to process this information and present it in a comprehensive clinical format; to treat disorders effectively when diagnosed; to monitor treatment and evaluate its efficacy[1]. Therefore cost-effective diffusion telemedicine will not only reduce the cost of health care, but also enhance the productivity of health workers.

Health care is a special commodity in emerging economies, in both economic and social terms. South Africa is no exception. For many countries, it is only a problem on a priority scale dominated by poverty, a growing population and rural to urban migration[1]. About 70-80 percent of population in most African countries lives in rural areas[2]. Although, South Africa is economically advanced compared to other African countries, it still has a huge rural population. This rural population receives inadequate health services, because most health services are concentrated in urban areas and cities. The health care gap between preventive health and curative medicine is largely suspected as the source of chronic diseases in rural areas of South Africa[3].

The advent of telemedicine services, which is the use of telecommunication and information technologies to provide health care services[4], has brought hope of providing health care services to the rural population. In South Africa, the

* Corresponding author:

michael.kachienga@up.ac.za (Michael Ogembo Kachieng'a)

Published online at http://journal.sapub.org/phr

Copyright $(C) 2012$ Scientific \& Academic Publishing. All Rights Reserved benefits of telemedicine services have not been fully realized because of infrastructural constraints such as the lack of bandwidth and telephonic services. This is mainly due to the lack of telecommunications infrastructure that is required as a platform for telemedicine care in rural areas. Other technical issues stunting effective utilization of telemedicine services include unreliable power supplies and frequent load shedding in rural areas.

Effective management of technological infrastructures and health care resources is also lacking in rural clinics and hospitals because competent health workers prefer to work in urban areas and cities[3].

\section{Making Clinical Sense of Telemedicine}

Telemedicine broadly refers to the use of communication and information technologies for the delivery of preventive and clinical care. The more definitions of telemedicine are broader and more inclusive. In the modern health care delivery environment, the term telemedicine more correctly describes the direct provision of preventative and clinical care via telecommunication systems - facilitating diagnosis, treatment or a follow up with a patient at a distant. In past one decade, telemedicine has been utilized by public and private health providers in a growing number of specialties such as oncology, radiology, surgery, psychiatry and home-based health care[4,5]. Telemedicine can be simple as two clinical experts discussing a patient's case on phone or as complicated as using satellite system to broadcast a consultation between clinical consultant and a doctor in two distant locations - city and rural hospitals, using videoconferencing system. Telemedicine allows patients to obtain regular health care services from a primary health provider 
or clinic without having to leave their local communities, thus ensuring continuity of care and periodic follow up as dictated by treatment protocol. The goal of diffusion of telemedicine and other health technologies in the health care system value chain is to enhance cost-effectiveness and increase efficiency of health care delivery[5].

\subsection{Rural Telemedicine Services}

The rapid advances in telecommunication technologies has led to the development of integrated service digital network (ISDN) that can provide an array of services including video traffic to enable videoconferencing that can be used to provide face-to-face consultation between a patient at a clinic and a physician in a hospital several kilometres away. However the lack of Integrated Service Network (ISDN) connections and the high cost associated with such technologies have made ISDN inaccessible to most rural communities in South Africa, thus making video conferencing for face-face consultation using telemedicine impossible[3].

Communication networks can be broadly defined as an arrangement of hardware and software that allow users to exchange information[4]. The information exchange may be voices, sounds, graphics, pictures, video, text, or data, among users. Traditionally, the most common types of communication networks: the telephone network for voice transmission, and a computer network and peripherals. The advancement in communications and information technologies has resulted in new communication networks merging the capabilities of both telephone and computer networks into one network. This has in turn paved the way for development of high-speed networks that are able to transmit high quality, full-motion video.

A communication network consists of a set of nodes that are interconnected to permit the exchange of information. These nodes can be classified as either terminal nodes, or as communication nodes. The terminal node has terminal entities such as phone sets, computers, printers, files servers, or video monitors, whereas the communication node, on the other hand has communication devices such as switches, routers, or repeaters. The terminal nodes generate or use information transmitted over the network, while the communication nodes transport the information but do not generate or use the information[4].

The nodes can be a few meters to many kilometers apart. To link the nodes a communication channel is used, which may be a transmission line, an optical fiber, a radio link, or a light wave in free space. For rural areas the nodes can be a considerable distance apart; for example, a network that can enable clinics and the local hospital to exchange information can have nodes that are more than 50 kilometers apart. The other problem with rural communication networks are the different types of terrains over which the communication equipment or cables have to be deployed. Most rural areas are characterized by mountainous terrains, valleys, forests and rivers, which can make the use of other types of transmission media, such as transmission lines and fiber optics rather difficult.

The convergence of telecommunication and communication technologies forms the backbone of telemedicine care services. Telemedicine care is considered as one of the solutions to the problem of health care delivery in rural areas by improving communication capabilities and providing convenient access to up-to-date information on-line, consultations and other forms of multimedia support[6].

\section{Rural Area Network}

The rural area network (RANET) is a multi-service packet-based communication network, which can be used to provide an array of services to rural areas. The system is autonomous and tailored to provide performance matching the requirements of different applications. The RANET system architecture is designed to deliver integrated services to both wired and fixed-wireless networks. It is an open system for converged networking as shown in Figure 1, and consists of the following four elements: services and applications network; core network; broadband access network; and user terminals. The architecture ensures that these functional elements are seamlessly interconnected and facilitates connections of additional networks, such as Internet, and the traditional telephone service network, to the core network.

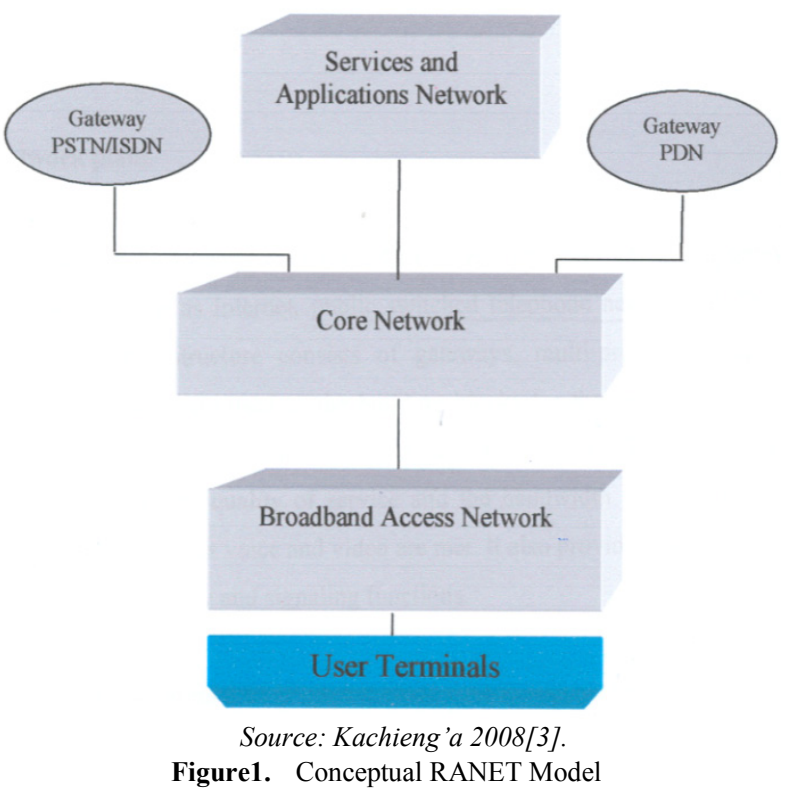

The architecture also enables, inter alia, the following[4]:

Packet services: As a data transport, it should provide carriage for video, voice and data applications with an appropriate quality of service for each. It should support the existing Internet Protocol (IP) applications and new value-added services.

Real-time communication: These are interactive real-time voice and video communication.

Multicasting services: This allows for efficient delivery of a given information stream of multiple recipients at the same 
time, which is important for continued medical education (CME).

Network management: These are supervisory functions, such as monitoring, and maintenance, to keep the network operating efficiently and effectively.

Unified messaging: This involves the integration of voice, fax, and e-mail messages on single IP-based message repository.

The core network plane links the users with feature servers and internet-working gateways to external networks such as internet, public switched telephone network (PSTN) and other networks. Its infrastructure consists of gateways, multi-protocol router and multilayer switches, which are used as the building blocks for the network. The core network is build around IP-packet based core to support the multiple needs of users and to reduce the cost of network operation[3].

\subsection{Models of Rural Telemedicine Service Design}

There are two models that can be used to provide rural telemedicine: local and open systems. These models are shown in Figure 2 and Figure 3, respectively.

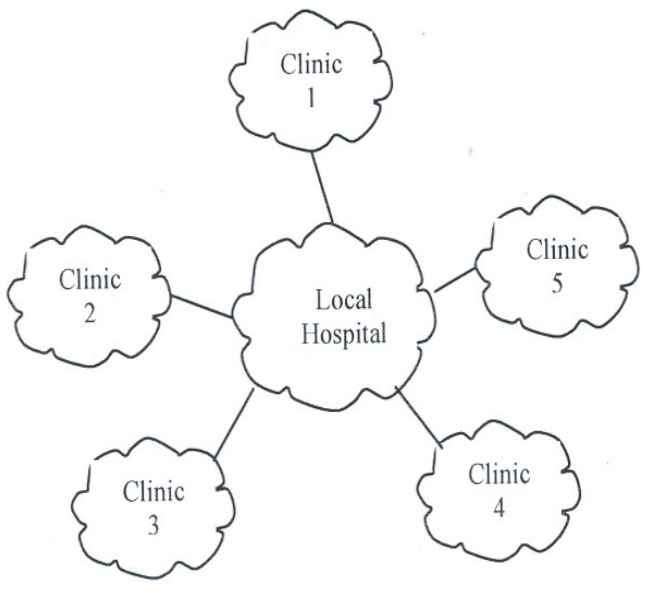

Source: Kachieng'a 2008[3]

Figure 2. Local rural telemedicine system

The local system is a network that is constituted of a local hospital and a number of surrounding clinics. This model, though simple, tends to confine the clinicians to the limited expertise in their area. A system based on local configuration was launched in December 2002 in the mountainous Qumbu district in the Eastern Cape. The project is a joint venture between the Council for Science and Industrial Research (CSIR) and Tsilitwa community. It uses ICT to link Tsilitwa clinic to Sulenkama community hospital, which is about 20 kilometers away from the clinic. It is an autonomous system based on wireless Local Area Network (LAN), and provides wireless connection to clinics, schools, and police station using an Internet Protocol (IP) based Private Automatic Branch Exchange (PABX) and a simplex wireless video link between the clinic and the hospital. The telephone and the video links between the clinic and the hospital enables the physician at hospital to see the condition of the patient at the clinic, so that the medical staff at the clinic can be advised of appropriate intervention[4].

Figure 3 shows an open rural telemedicine system. The system is designed to have direct links with other secondary and tertiary medical institutions to enable direct consultation between clinician and physician or specialist in any other medical institution, be it locally, or in the city. The competitive advantage of this model is that the rural clinic health professional has a broader spectrum of medical experts at his/her disposal to consult from all institutional sites in the network. The open rural telemedicine system provide multi-consultation access between telemedicine health providers and specialists based at regional and city health facilities, thus enhances the efficiency of delivery of services.

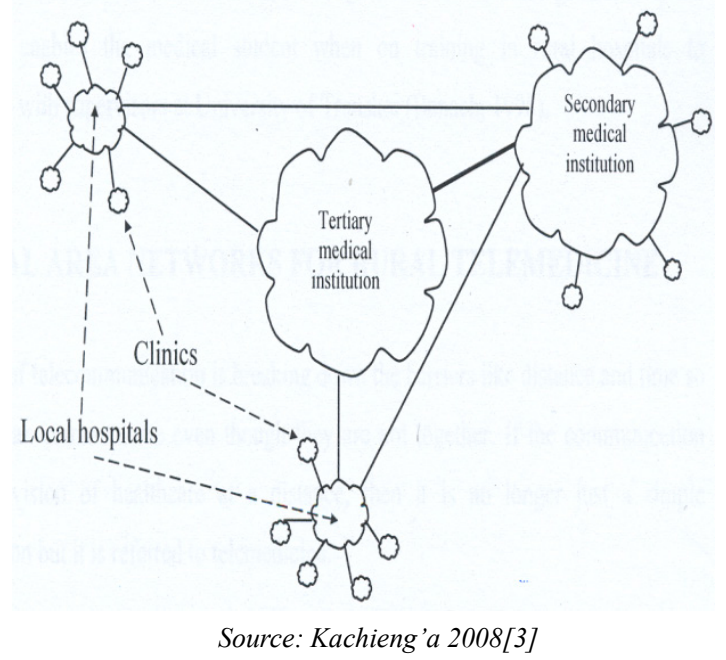

Figure 3. Open rural telemedicine system

\subsection{Health Technology Infrastructure Investments}

Strategic planning and management of technological investments in health care systems, hospitals and clinics are the most difficult challenges facing health policy makers and planners in South Africa. Although the public health care system is far from being homogenous, it is evident that two large groups of technologies are considered simultaneously namely, technology for primary health care services and hospital-based technologies for curative services. The effective management of the two groups of technologies will lead to competitive delivery of health care services - higher outputs at lower costs and better access to health care services. It is therefore imperative that investments in health technology infrastructure should take into account technological relationships between various levels of health care delivery services to ensure the cost-effective utilization of technological investments and better return on investment.

\subsection{Telemedicine Technology Diffusion Management}

Health technology management has been defined as an accountable, systematic approach to ensuring that cost-effec tive, efficacious, safe, and appropriate equipment is available to meet the demands of quality patient care[6]. Modern health care institutions, even in emerging economies such as 
South Africa, are increasingly embracing technological innovations in health care. The uptake of advanced health technology in health system is considered as an economic imperative for a better health care delivery and coverage[8].

Technological innovations in $21^{\text {st }}$ century have reshaped the field of medicine and the delivery of health care services. Recent advances in health technology have provided a wide range of diagnostic, therapeutic, and rehabilitative tools and instruments that are now routinely used in the cure of specific diseases and illnesses. It is within such a process that the modern hospitals have evolved as technologically sophisticated health care facilities serviced by technologically specialized personnel. The changes have contributed significantly to the quality of health care provided by various health facilities, from teaching hospitals to rural clinics; but have also brought new challenges in health technology management in South Africa[8].

Telemedicine is unique, in that it has the capability to cross geographical, political, social and cultural barriers within the health sector. With comparatively fewer resources, extended outreach and yet contained health cost, several essential health care services can be provided to a large number of the geographically scattered rural population. A key future of telemedicine, services is the use of multimedia peripheral devices that include electronic versions of standard diagnosis and examination tools as well as other sense extending implements like cameras, document stands, derma scopes and microscopes. The multimedia peripheral devices enable the clinician to better approximate an on-site physical examination.

Another key future of telemedicine is the use of multimedia peripheral devices that include electronic versions of standard diagnosis and examination tools as well as other sense extending implements like cameras, derma scopes and microscopes. The multimedia peripheral devices enable the clinician to better approximate an on-site physical examinations.

The management of telemedicine technology cannot be effective when confined within organizational boundaries. The management of telemedicine technology is inter-organizational endeavour and the exploitation of its full utility requires management strategies extending across organizational boundaries. Factors such as maintenance budgetary allocations, technical support services are very critical for operation of efficient telemedicine services.

It is important to note that telemedicine services be designed to add-value to other health care services and are not intended to exclusive[8]. The cost-effectiveness of telemedicine services depends on organizational capacity to facilitate effective integration on hospital-based and outreach health services.

Managing both technical and health aspects of telemedicine services have been major challenges to both government and donor organizations who normally fund telemedicine projects. Providing rural population with telemedicine services through improved video quality can facilitate access to health care services while, at the same time reducing the cost of care, through timely service delivery and reduction of unnecessary hospitalization.

Management of telemedicine technology diffusion cannot be effective when confined within organizational boundaries. Telemedicine is inter-organizational and capitalization of its full utility requires technology management extending across organizational boundaries. Factors such as technology base and technical support services, attitude of clinical staff and organizational management commitment to the delivery of telemedicine services are very critical for efficient operation and coordination. Effectiveness of telemedicine services depend on organization coordination and integration with other clinical services.

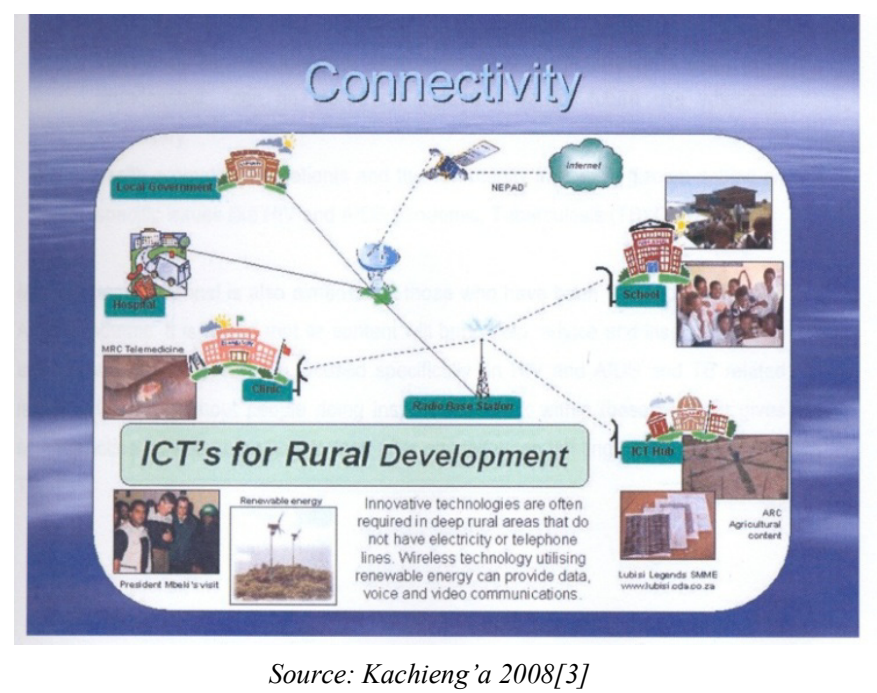

Figure 4. Typical telemedicine infrastructure

Managing both technical and health care aspects of telemedicine competitively has been a major challenge to both government and other health care providers. Providing rural telemedicine systems with improved video quality can improve health care services access, while at the same time reducing cost of care, through timely service delivery and reduction of unnecessary hospitalization.

\subsection{Financing Telemedicine Projects}

Historically, most telemedicine projects have been financed through donor funding or bilateral grants from foreign governments. Normally funding covers the project period ranging between $2-5$ years. Within the project period telemedicine services are funded through a budget line separate from the public health services budget[10]. On completion of the project period all telemedicine services assets are handed over to the host Government. Thereafter, both capital and operational expenditure are expected to come from the public health care services budget.

Operational costs such as bandwidth and telephone bills are supposed to come from the maintenance equipment budgets from the government health budget. The maintenance budget for public health technological assets is normally inadequate, thus curtailing the delivery telemedicine 
services in South Africa. The cost of ownership of telemedicine equipment and associated technical services has not been factored into the public health system to promote efficient and cost-effective telemedicine services in the rural areas of South Africa.

\subsection{Telemedicine Health Service Providers}

Although majority ( $80 \%$ ) health care providers (12 people -7 medical personnel and 5 medical managers - doctors) were interviewed at various sites were appreciative of investments in telemedicine technologies to provide high quality health care services to rural communities of South Africa, they also highlighted lack of sustainable technical and budgetary support. They believe that the introduction of telemedicine in the health care delivery value chain will enhance and generate efficiency in the traditional health care referral system and reduce costs of health care services delivery. They stated that apart from cutting costs of health care, the injection of telemedicine in the health care delivery value chain has the potential of improving health care outcomes. The major draw-backs in cost-effective delivery telemedicine services are poor technical support, inadequate operational budgets and training of health service providers.

According to five senior medical officers interviewed during the survey, the lack of adequate facility-level budgetary support and lack of trained medical staff with adequate skills to run telemedicine services. Most of regular medical staff views telemedicine as an add-on service to the regular health care services and therefore not the main focus for their operations. In most cases there is no operational budget-line for telemedicine services. Sometimes the budget for telemedicine is lumped together with maintenance budget. According to doctors, telemedicine services can becost-effe ctive if it is incorporated into main stream health system.

\subsection{Telemedicine Patients Management}

Patients interviewed at the 11 sites within the Eastern Cape, South Africa, showed a lot appreciation for the availability of telemedicine services in rural areas. However, they complained about the unpredictability of services, due to power failure or load shedding and lack of bandwidth due to non-payment of telephone bills. They also indicated that when the services unavailable due to technical problems, they are made aware until the visit the clinic. Such information can be passed to patients through mobile phone to avoid travelling to clinics. Most of rural residents are peasant farmers and poor, and cannot afford transport costs to hospitals. Telemedicine service in rural areas of South Africa is a major boost to the delivery of public health care services, but management of the services still remains a challenge.

\section{Discussion}

A greater commitment by the South African Government to address disease burden in rural areas has resulted in public and private sectors investments in the development and diffusion competitive health technologies such as telemedicine, new vaccines, diagnostic and therapeutic instruments to support prevention and treatment of these diseases. Telemedicine offers both preventive and curative services in the health care delivery value chain. Therefore effective diffusion and utilisation telemedicine will not only reduce the cost health care services, but also offer a wider access to health services to the isolated rural populations. The World Health Assembly has acknowledged that telemedicine could play an important role in providing access to health for population at risk worldwide, especially in Africa[11].

Although telemedicine services can be technologically independent from other health care services, they remain value-adding services to main stream health care services. Sustainability and success of telemedicine services depend on their strategic integration into other health services provided by hosting institutions. To be efficient andcost-eff ective, it must be administratively and technologically integrated with other health care services provided by hosting hospitals and clinics. Public health planners have always assumed that because telemedicine is a value-add service, they took for granted that medical and technical staff at the hospital and clinics will embrace the introduction of telemedicine services. That has not been the case according to the survey conducted in the Eastern Cape[8]. The components of the evaluation framework are shown in figure 5 . The evaluation of telemedicine projects covered: clinical and technical management, technical support and clinical adoption. The objective was to tease out success factors to ensure success and sustainability for telemedicine services.

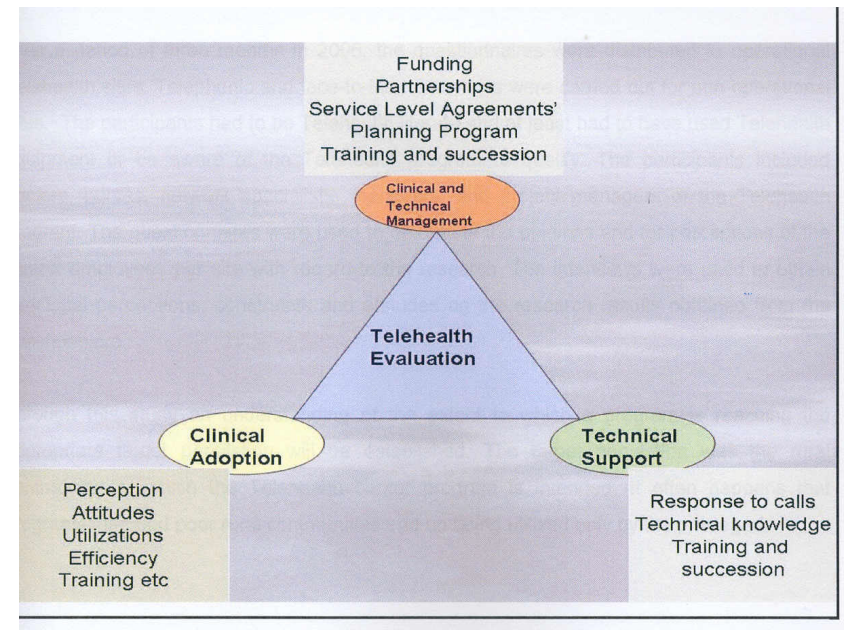

Source: Kachieng'a 2008[3].

Figure 5. Evaluation framework

Most rural hospitals and clinics are medically understaffed and technically under-resourced; therefore the introduction of new services becomes a burden rather than a relief for the staff. There is also a need for continuous training of both medical and technical staff because of regular upgrades. High productivity in telemedicine services depends of seamless collaboration and coordination of medical and technical staff. Most initial funding of telemedicine services 
tends to be project-based, while maintenance budgets tend to be institution-based. Rural hospitals and clinics have budgetary constraints leading to inadequate support of telemedicine services. Further more, telemedicine services have also been hampered by frequent power failures and load shedding in rural areas. Telemedicine can effectively alleviate health services problems in rural areas if properly coordinated, strategically managed with adequate budgetary support.

The observations and insights from this study can provide policy makers and health planners with data for improving health technology investments strategy to support accessible and cost-effective health care services delivery in rural South Africa.

In the final analysis, the study shows that the major constraints in delivery telemedicine services are the management of the technological systems, providing sufficient operating budgets and adequate training of health service providers.

\section{Conclusions}

The demand for improved and accessible health care services by rural communities in South Africa is growing rapidly. Access to basic education and health care services are becoming human right issues. Furthermore health is one the major driver of economic growth and social change. It is within this strategic framework that the South African government investment in telemedicine projects is aimed at providing outreach health care services to rural populations.

Providing appropriate and effective health care services in rural areas remains a major challenge for the South African Government. Telemedicine could improve delivery of health services but requires coordination and strategic management of all associated technologies; and adequate operational budgetary support. There is also a need to improve technical infrastructure including stability of power systems, provision of adequate bandwidth for voice, image and data.

It is important that the technical support and system maintenance be adequately funded to ensure sustainability of telemedicine services in rural areas. Although health technology acquisition budgets are normally catered for, it is evident budgetary allocation for maintenance and consumables are inadequate to sustain telemedicine services. Further more, technicians and engineers are not readily available in rural health institutions to ensure adequate technical support of telemedicine services. In addition, power systems in rural areas are not only inadequate but also unreliable, leading to the under utilization of telemedicine technologies. There is also need for continuous training of both medical and technical staff on the utilization of telemedicine technologies due continues technological innovations in the health sector.

The study shows that, for successful diffusion of telemedicine services in South Africa, it is of paramount the budgetary support for maintenance and technical services be competitive and sustainable. It is also crucial that health policies pertaining to telemedicine in particular and e-health in general be formulated and implemented to broaden the coverage and enhance delivery of health care services delivery.

The recent advancement in ICT and telemedia technologies, innovations in wireless systems and widespread penetration of broadband in South Africa have increased the economic attractiveness of telemedicine services as a viable alternative method for public health care services delivery. Telemedicine has the potential to close the gap between primary health and curative care. It is evident that further innovations in telemedicine systems will have potential to address critical issues in public health care services such as access, coverage and cost. Evidences from other emerging economies show that effective planning and deployment of health technologies are the cost-effective methods for ensuring equitable delivery of health care services to the rural population of South Africa.

\section{REFERENCES}

[1] M.O. Kachieng'a, "Health technology assessment in Sub-Saharan Africa: A Cross-National study of Kenya and South Africa." Doctoral Thesis, University of Cape Town, South Africa 1999.

[2] S. McClelland and A. Berendt, "Rural Africa, Making Connection." Supplement to Telecommunication International, April 1998.

[3] M.O. Kachieng'a, "Technological Innovations in Health Care: Challenges of Managing Technology in South Africa”. IEEE International Engineering Management Conference (Europe -Portugal; June 2008.

[4] P. Malindi, "Methods for Providing Rural Telemedicine with Quality Video Transmission.” Doctoral Thesis, Cape Peninsula University of Technology 2006.

[5] D.A. Perednia, A. Allen, Telemedicine technology and clinical applications. JAMA February 8, 273(6): 483 - 8, 1995.

[6] D. McCarthy, "The Virtual Health Economy: Telemedicine and the Supply of Primary Care Physicians in Rural America.' American Journal of Law \& Medicine, Vol. 21, No 1.1995.

[7] Emergency Care Research Institute (ECRI), "Technology Management: Preparing Your Hospital for 1990s." Health Technology, 3:111-115. 1989.

[8] M.O. Kachieng'a, “An African Safari in Health TechnologyFrom Cape Town to Nairobi in 10 Days," South African Medical Journal, 92(5):344-345. 2002.

[9] M.A. Mbanga, "Evaluation of Telemedicine Centers in the Eastern Cape Province, South Africa." Masters Thesis in Technology Management. University of Pretoria, 2006.

[10] D. Brantly, "Innovation, demand and investment in Telehealth." Report Review, 2004.

[11] N.E. Lugn, "Global health care - bridging the gap." Journal of Telemedicine and Telecare 12(3): 109-10, 2006. 